\title{
Automating the Use of Web APIs through Lightweight Semantics
}

\author{
Maria Maleshkova ${ }^{1}$, Carlos Pedrinaci $^{1}$, Dong Liu ${ }^{1}$, and Guillermo Alvaro ${ }^{2}$ \\ 1 Knowledge Media Institute (KMi) \\ The Open University, Milton Keynes, United Kingdom \\ \{m.maleshkova, c.pedrinaci,d.liu\}@open.ac.uk \\ 2 Intelligent Software Components (iSOCO). Madrid, Spain \\ $\{$ galvaro\}@isoco.com
}

\begin{abstract}
Web services have already achieved a solid level of acceptance and play a major role for the rapid development of loosely-coupled component-based systems, overcoming heterogeneity within and between enterprises. Current developments in the world of services on the Web are marked by the proliferation of Web APIs and Web applications, commonly referred to as RESTful services, which show high potential and growing user acceptance. Still, despite the achieved progress, the wider adoption of Web APIs is hindered by the fact that their implementation and publication hardly follow any standard guidelines or formats. REST principles are indeed a good step in this direction but the vast majority of the APIs do not strictly adhere to these principles. As a consequence, in order to use them, developers are obliged to manually locate, retrieve, read and interpret heterogeneous documentation, and subsequently develop custom tailored software, which has a very low level of reusability. In summary, most tasks during the life-cycle of services require extensive manual effort and applications based on existing Web APIs suffer from a lack of automation.

This tutorial introduces an approach and a set of integrated methods and tools to address this drawback, making services more accessible to both experts and non-expert users, by increasing the level of automation provided during common service tasks, such as the discovery of Web APIs, their composition and their invocation. The tutorial covers i) the conceptual underpinnings, which integrate Web APIs with state of the art technologies from the Web of Data and Semantic Web Services; ii) the presentation of an integrated suite of Web-based tools supporting service users; iii) and hands-on examples illustrating how the tools and technologies can help users in finding and exploiting existing Web APIs.
\end{abstract}

\section{Description}

The tutorial is entirely driven by and supported by current developments on the Web, and will familiarise the participants with innovative yet applicable tools and technologies that could directly be integrated in Web developers daily activities. It provides both key background information as well as an approach for addressing some of the main challenges faced when using Web APIs. We introduce an integrated set of tools, which support the automated use of APIs: 
- Web API Annotation via SWEET [1] is a Web application that supports users in creating lightweight semantic descriptions of Web APIs by enabling the marking of service properties within HTML documentation and associating these with semantic annotations.

- Storage and discovery via iServe [2] is a public registry of semantic Web services, which unifies service publication and discovery on the Web through the use of semantics. iServe imports the semantic descriptions of Web services conforming to heterogeneous formalisms (hRESTS, MicroWSMO, SAWSDL, and OWLS) and publishes them as RDF triples on the Web of Data using a lightweight service model.

- Consumption via the generic client of an invocation framework that provides a unique entry point for the invocation of most Web APIs that can be found on the Web. The framework relies on non-intrusive semantic annotations of HTML pages describing Web APIs, in order to capture both their semantics as well as information necessary to carry out their invocation.

\section{Tutorial Modules}

Introduction (morning session): This presentation provides required background information, short overview of the key issues and concepts related to the usage of Web services, best practices while dealing with Web APIs, and the support offered through applying lightweight semantics.

- Current trends and developments in the world of services on the Web

- Problems and challenges faced when using Web APIs

- Current approaches for discovering and invoking Web APIs

- Best practices with Web APIs and use of lightweight semantics

Hands-on session part I (afternoon session): Using the presented approach and tools to support Web API search and discovery.

- Performing service search without tools support

- Web service annotation

- Web service search and discovery

Hands-on session part II (afternoon session): Using the presented approach and tools to support service invocation.

- Invoking services

- Service monitoring

- Example applications and implementations

Slides from the training session can be viewed at http://www.slideshare.net/ mmaleshkova/automating-the-use-of-web-apis-through-lightweight-semantics.

\section{References}

1. SWEET, http://sweet.kmi.open.ac.uk

2. iServe, http://iserve.kmi.open.ac.uk 